\title{
CASOS DE MARKETING COMO VINCULACIÓN UNIVERSIDAD-EMPRESA.
}

\author{
Manuela Camacho Gómez*.
}

\author{
Camacho-Gómez M. Casos de marketing como \\ vinculación universidad-empresa. Hitos de Ciencias \\ Económico Administrativas 2012;18 (50):9-18. \\ RESUMEN
}

El marketing comoárea funcional de las organizaciones, ha superado el diseño creativo por acciones estratégicas que favorezcan la imagen corporativa de las empresas y las instituciones. Esto se logra mediante, identificación de mercados, selección de segmentos bien definidos, a quienes se atenderán con acciones de creatividad calculada que permitan posicionamientos que signifiquen, en la medida de lo posible, una amplia participación de mercados.

Desde esta perspectiva, el marketing se traduce en volúmenes de ventas acordes con la capacidad productiva y el know how organizacional; la colocación de productos y servicios necesariamente se traducirá en retornos de inversión mercadológicos apropiados a los objetivos de las empresas e instituciones.

En este contexto, la vinculación universidad-empresa, tiene en los casos de marketing una herramienta didáctica apropiada para formar competencias directivas y gerenciales, desde la empresa para la empresa.
Camacho-Gómez M. Cases of marketing as university-business link. Hitos de Ciencias Económico Administrativas 2012;18 (50):9-18.

\section{ABSTRACT}

Marketing as a functional area in the organizations has exceeded the creative design for strategic actions to promote the corporate image of companies and institutions. This is achieved by market identification, selection of well-defined segments that will be met with creative calculated actions that allow positions to mean, as far as possible, a large market share.

From this perspective, marketing is understood as sales volumes according to the production capacity and the organizational know-how. The product and services placement necessarily will mean marketing investment returns appropriated to the objectives of companies and institutions.

In this context, the university-business link has, in the cases of marketing, a suitable teaching tool to develop policies and management skills from the company to the company.
Palabras clave: Marketing. Estudios de Caso. Estudiantes de marketing.
Key words: Marketing. Case Studies. Students of marketing .

\section{DIRECCIÓN PARA RECIBIR CORRESPONDENCIA: Correo electrónico: manuela.camacho@ujat.mx}

\footnotetext{
* Doctora en Educación Internacional. Profesora-Investigadora. División Académica de Ciencias Económico Administrativas. Universidad Juárez Autónoma de Tabasco (UJAT).
} 
as tendencias en las escuelas de negocios se enmarcan en una corriente educativa progresista que insta a la formación integral de los estudiantes, en estas acciones, se encuentra una mayor relación con los mercados laborales y un vínculo más estrecho entre la universidad-empresa.

En este contexto, una forma de vincular a los alumnos con los sectores productivos es través del análisis o resolución de Estudios de Casos empresariales. Bajo estas condiciones, las empresas comparten sus historias y situaciones susceptibles de ser descritas metodológicamente estructuradas en formas de casos, material que llega a los estudiantes a manera de «simulador de negocios escrito» (Camacho, 2011).

Las universidades reconocen que las exigencias del mercado de trabajo requieren de profesionistas competentes, capaces de tomar decisiones, enfrentar obstáculos, ejercer la crítica, expresar puntos de vista, escuchar a otros y coadyuvar con estrategias creativas al crecimiento y rentabilidad de las empresas y consolidación de instituciones, estas competencias son desarrolladas a través del método de casos, con temas de marketing.

Considerando la dinámica de las escuelas de negocios, la Licenciatura en Mercadotecnia (LEM) de la División Académica de Ciencias Económico Administrativas (DACEA) de la Universidad Juárez Autónoma de Tabasco (UJAT), es una carrera propicia para utilizar la técnica didáctica del caso.

\section{Antecedentes.}

El enfoque centrado en competencias trata de transformar los contextos no solo a las personas, ya que éstas se forman intersubjetivamente y propicia la formación centrada en la resolución de problemas como la vía idónea que posibilita el cuestionamiento y la problematización de los saberes, creencias y valores que se poseen y posibilitan asumir nuevas maneras de ver la realidad desde nuevas aristas al intervenir en ellas (Rodríguez, 2000).

Bajo esta perspectiva, los Estudios de Caso tienen un objetivo didáctico para fortalecer las competencias directivas y gerenciales de los estudiantes. Aunque los casos no son transmisores de conocimiento (Ogliastri, 1993), si estimulan su generación, por esa razón, responden cabalmente a las exigencias globales en pos de un aprendizaje significativo donde el ésta técnica es de gran relevancia.

Los organismos supranacionales e intergubernamentales, dictan sus directrices hacia un proceso de enseñanza-aprendizaje significativo con didácticas acordes como el método de caso que es el más usado por diversas universidades y escuelas de negocios nacionales e internacionales. Es el modelo educativo utilizado por universidades prestigiadas como Harvard en los Estados Unidos, el INCAE Business School en Costa Rica y Nicaragua y en México por el Instituto Panamericano de Alta Dirección de Empresas (IPADE) y el Instituto Tecnológico de Estudios Superiores de México (ITESM).

En México, el uso del método del caso, tiende a ser privilegiado en las universidades privadas, como el Instituto Tecnológico de Estudios Superiores de Monterrey (ITESM), el cual cuenta con el Centro Internacional de Casos (CIC), además de liderar a la Asociación Latinoamericana de Casos (ALAC) como miembro activo del World Association for Case Method Research \&Application (WACRA). Sus estudiantes son formados con casos y sus profesores los escriben y aplican en clases.

Por su parte, el Instituto Tecnológico Autónomo de México (ITAM), tiene dentro de sus métodos de estudio la resolución de diversos casos prácticos en todas las materias del área gerencial y también constituye una forma de titulación en el posgrado de Administración y Dirección Internacional, en esta institución, son los estudiantes posgraduados quienes escriben casos como parte de un trabajo grupal que es tomado como tesis y cuya defensa se hace ante un jurado compuesto por especialistas en el tema. Algunos de estos trabajos son utilizados en clases.

El Instituto Panamericano de Alta Dirección de Empresas (IPADE) cuenta con una base de casos prácticos de aplicación internacional distribuido por el IESE de España. Muchos de los casos de empresas líderes nacionales han sido escritos por profesores del IPADE.

Organismos nacionales como la Asociación Nacional de Facultades y Escuelas de Contabilidad y 
Administración (ANFECA) en sus asambleas anuales han realizado acciones para fortalecer el uso del método del caso y han convocado durante 7 años consecutivos al concurso nacional de casos de contaduría y administración, para fomentar la escritura de casos por parte de profesores de las diferentes instituciones de educación superior (IES) que se encuentran adheridas a esta asociación. En este tenor, la ANFECA ha creado su propia metodología para la escritura de casos.

\section{El método de caso.}

El método de casos es una técnica didáctica apropiada para la formación y actualización en las ciencias económico administrativas, sin embargo, su uso requiere de competencias especializadas por parte de los profesores y una preparación previa de los usuarios para potenciar las ventajas del método. El método del caso en la enseñanza o la instrucción, describe una situación real de una organización empresarial, institución o personas, en un contexto y en un periodo dado (Camacho, 2011).

Un caso es una forma de enseñanza donde los estudiantes deben involucrarse para aprender (Naumes, 2006). Esto quiere decir que, se trata de un hecho real o una situación que atraviesa alguna persona u organización en un momento dado de su historia, la cual puede ser favorable o desfavorable (Ogliastri, 1999; CIC n/d).

La disciplina, el formato, la metodología y la intención didáctica, orientan los pasajes empresariales que se retratan en un caso, los cuales usualmente son planteados de forma fluida y ricos en datos, el estilo de escritura puede provocar un grado de entusiasmo diferente (Camacho, 2011).

Los casos se clasifican según su enfoque, magnitud y estructura, según Lundberg \& Rainsford (2001), los casos se pueden agrupar en nueve tipos: Iceberg, Incidente, llustrativo, Central, Diálogo, Aplicación, Dato, Asunto y Predicción.

Por su extensión, los casos se consideran mini (de dos párrafos a 1 página) y casos en extenso (de dos hasta 30 páginas), estas consideraciones dependerán del segmento al que vayan dirigido los casos, los objetivos pedagógicos que pretendan alcanzar y sobre todo el enfoque metodológico bajo el cual sean escritos.

\section{Casos en la DACEA-UJAT.}

Los Casos requieren de la aplicación de un proceso de investigación en un primer momento y en de una metodología probada para escribirlos. En este sentido, la contextualización de los Casos puede ser un componente clave para la formación de competencias en estudiantes de pregrado.

En laUJAT, la primera carrera centrada en competencias es la Licenciatura en Mercadotecnia (LEM) donde el plan de estudios contempla técnicas didácticas que formen competencias directivas y gerenciales en el estudiante, entre ellas se encuentra el método de casos. En este esquema, el método del caso potencializa sus ventajas ya que se le percibe como un modo de enseñanza apropiada para los estudiantes de la LEM.

En este esquema, el profesor-investigador puede asumir el papel de facilitador en el uso del método o bien ser el escritor de casos, para lo cual debe plantear con claridad el objetivo pedagógico fundamental que se espera de cada caso. Bajo este método los alumnos aprenden sobre la base de experiencias y situaciones de la vida real, permitiéndoles así, construir su propio aprendizaje en un contexto que los aproxima a su entorno. Este método ayuda a desarrollar la participación activa, las habilidades analíticas, promueve la interacción con otros y la comunicación efectiva tanto oral como escrita derivado de los procesos colaborativos de discusión de los hechos reflejados en el caso (Camacho, 2011).

\section{Reflexiones sobre la problemática.}

El método de casos en la Licenciatura en Mercadotecnia de la UJAT se utiliza de manera aislada y la conducción del profesor no es acorde con los requerimientos del método, esto se debe en parte, a que no existen profesores de casos, a que los casos que se utilizan son los denominados mini-casos - de 1 a tres páginas con preguntas de reflexión- que se localizan en libros de texto y a que la mayoría de ellos corresponden a empresas transnacionales y las situaciones que se describen en el caso corresponden a contextos culturales y geográficos distantes y desconocidos para los estudiantes. 
Esta situación también se debe a que la DACEA no dispone de casos propios que pudieran coadyuvar al desarrollo de habilidades directivas y gerenciales de los estudiantes de LEM en contextos de alta familiaridad para ellos. El no contar con casos conduce a que cuando aisladamente se use esta técnica didáctica, se corra el riesgo de que éstos probablemente se encuentren en un contexto cultural ajeno, o que se trate de situaciones que no se vinculen directamente con los objetivos de las materias de la licenciatura. Bajo estas condiciones, el estudio de casos puede ser contraproducente o bien, podría estar siendo sub utilizado.

Como respuesta a esta situación, la DACEA-UJAT a través del CuerpoAcadémico de Estudios Estratégicos para Negocios e Instituciones (CAEENI), se encuentra desarrollando investigaciones empresariales para conocer la aplicabilidad o uso del método del caso en las universidades de Tabasco que imparten la Licenciatura en Mercadotecnia.

Derivado de los resultados de estos estudios, se podrá conocer la potencialidad del método y la demanda de casos prácticos provenientes de investigaciones con empresas locales y regionales que compartan una parte de su historia que a su vez, denoten momentos cruciales de la organización, en situaciones de éxito o de desafíos. Estos periodos narrados por los empresarios permitirán una fuente de información confiable de donde se deriven casos en contextos geográficos y culturales conocidos por los estudiantes.

\section{La investigación.}

Uno de los proyectos que se encuentra realizando el CAEENI financiado por el Programa de Fortalecimiento Institucional de Cuerpos Académicos (PFICA), es el denominado «Diseño de materiales didácticos para la formación integral del Licenciado en Mercadotecnia», cuyos resultados preliminares muestran una alta aceptación por los casos prácticos. Los estudiantes entrevistados en tres universidades del Estado de Tabasco dejan ver su punto de vista acerca del uso y los beneficios del método del caso.

Para que estos hallazgos, en cuanto a la percepción favorable de estudiantes de la Licenciatura en Mercadotecnia se conviertan en productos terminados, es necesario contar con la participación de empresarios que estén dispuestos a compartir parte de su historia

TABLA I. PRIMEROS HALLAZGOS SOBRE LOS BENEFICIOS DEL MÉTODO DEL CASO.

\begin{tabular}{|c|c|c|c|}
\hline Pregunta & Universidad 1 & Universidad 2 & UJAT \\
\hline $\begin{array}{l}\text { Conocimiento del método de } \\
\text { casos. }\end{array}$ & $69 \%$ & $67 \%$ & $72 \%$ \\
\hline $\begin{array}{l}\text { Les gustaría utilizar el } \\
\text { método de casos. }\end{array}$ & $100 \%$ & $83 \%$ & $80 \%$ \\
\hline $\begin{array}{l}\text { Resolución de casos en sus } \\
\text { clases. }\end{array}$ & $81 \%$ & $100 \%$ & $85 \%$ \\
\hline $\begin{array}{l}\text { Número de casos resueltos } \\
\text { en su carrera. }\end{array}$ & $1-3 \operatorname{casos}(52 \%)$ & Más de 7 casos $(72 \%)$ & $1-3(45 \%)$ \\
\hline Fuente de casos & $\begin{array}{l}\text { Libros de texto }(50 \%) \\
\text { Revistas }(18 \%)\end{array}$ & Libros de texto $(82 \%)$ & Libros de texto (56\%) \\
\hline $\begin{array}{l}\text { Aplicación de casos } \\
\text { fortalece o desarrolla } \\
\text { competencias directivas y } \\
\text { gerenciales. }\end{array}$ & $67 \%$ & $61 \%$ & $45 \%$ \\
\hline $\begin{array}{l}\text { Los casos enseñan a tomar } \\
\text { decisiones. }\end{array}$ & $32 \%$ & $57 \%$ & $40 \%$ \\
\hline $\begin{array}{l}\text { Los casos permiten aplicar } \\
\text { conocimientos teóricos. }\end{array}$ & $39 \%$ & $43 \%$ & $31 \%$ \\
\hline
\end{tabular}

Fuente: elaboración propia con resultados preliminares de la investigación. 
en cierto periodo de su negocio. Después se precisará de elegir o apropiarse de una metodología probada para la descripción de los casos.

En este sentido, es necesario fortalecer las técnicas para el diseño de materiales aplicables a la didáctica y procurar la cualificación de los profesores que escribirán los casos, con lo que se estaría ejerciendo el vínculo universidad-empresa, es decir, una historia contada por el empresario, escrita por el profesor, utilizada por el estudiante, quien a su vez estará dotándose de las competencias requeridas por el mercado laboral, compuesto en su mayoría por empresas.

De esta manera la escritura y uso de los casos permitirán que los estudiantes de la Licenciatura en Mercadotecnia, sean formados bajo el enfoque de aprendizaje significativo, buscando que al mismo tiempo se garantice la calidad de la enseñanza, tal como lo privilegian las políticas educativas de la UJAT (UJAT, 2006). En consecuencia, se estarían utilizando materiales didácticos provenientes de las empresas para la formación del capital humano para la empresa, es decir, el vínculo apropiado entre universidad-empresa bajo un contexto de simulador de negocios escrito.

\section{REFERENCIAS}

Camacho, M. (2011). Casos de Marketing: 7 historias empresariales. UJAT: Villahermosa, Tabasco, México.

$\mathrm{CIC}$ (n/d). Escritura de casos. Recuperado en http:// cic.gda.itesm. $\mathrm{mx} / \mathrm{ClC} / \mathrm{index}$.php?ver=cursoEsc.php

Naumes, W. (2006). The art and craft of case writing. (2da) ed. United States of America.

Ogliastri, E. (1993). ¿Cómo escribir un caso? Monografías No. 35 Facultad de Administración. Universidad de los Andes. Bogotá, Colombia.

Ogliastri, E. (1999). Casos sobre casos: Experiencias con métodos de discusión en clase. Monografías. INCAE Business School. Costa Rica.

Rodríguez E. A.I. (2000). Estrategias de Marketing para un caso rentable: casos prácticos. España: ESIC.

UJAT (2006). Plan Estratégico de Desarrollo 20062016. México.
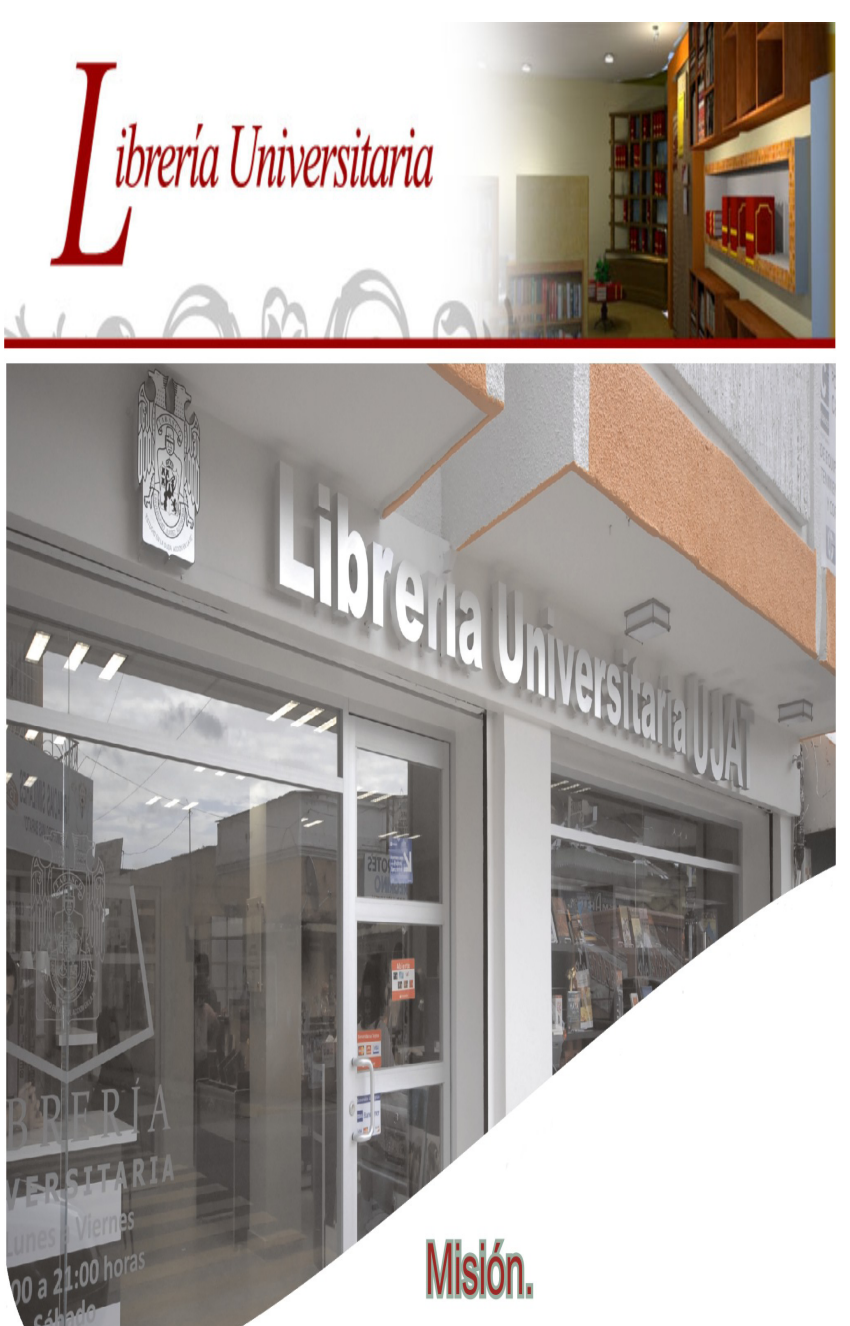

Difundir el fondo editoria UJAT, y los servicios inherentes con el afán de incidir en la formación integral del estudiante y de la comunidad, mediante la atención, eficiencia, amabilidad y cortesía en el servicio, ofreciendo publicaciones de calidad, con precios accesibles para satisiacer las necesidades de los clientes contibuyendo asi a la proyección nacional de nuestro quehacer tecnológico-cultural como aporte al desarirollo de la sociedad y la cultura latinoamericana.

\section{Visónal 2012}

Av. 27 de Febrero 626, Col, Centro, C.P. 86000 Villahermosa, Tabasco. Tel: 01 (993) 3124657

Lunes a Viernes de 09:00 a 21:00 Sábados de 09:00 a 13:00 www.ujat.mx libreria@ujatmx

Ser la mejor librería en la catecooría de universitaria en el estado, como centro difusor de la producción editorial universitaria y de otros fondos conformando un essacio cultural incluyente, destacando nuestros valores para vivir en una sociedad en amonía, paz s progresso, manteniendo el nivel de actualicad oue exila el momentio y con presencia a nivel nacionale intermacional.

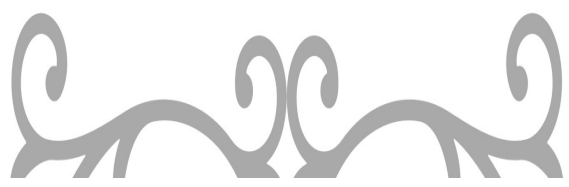




\section{Oferta Educativa de Licenciaturas}

Divisiốn Acade̋mica de Ciencias Biolögicas

Licenciatura en Biología

Licenciatura en Gestión Ambiental

Licenciatura en Ingeniería Ambiental

\section{Divisiő́n Académica de Ingeniería y Arquitectura}

Ingeniería Civil

Ingeniería Eléctrica y Electrónica

Ingeniería Mecánica Eléctrica

Ingeniería Química

Licenciatura en Arquitectura

División Acadëmica de Ciencias Sociales y Humanidades

Licenciatura en Derecho (escolarizado y sistema abierto)

Licenciatura en Historia

Licenciatura en Sociología

Divisiő́n Acadēmica de Ciencias Econốmico Administrativas

Licenciatura en Administración (escolarizado y a distancia)

Licenciatura en Contaduría Pública (escolarizado y a distancia)

Licenciatura en Economía

Licenciatura en Mercadotecnia

Divisiốn Académica de Clencias Agropecuarias

Ingeniería en Acuacultura

Ingeniería en Agronomía

Ingeniería en Alimentos

Licenciatura en Medicina Veterinaria y Zootecnia

Divisiōn Académica Multidisciplinaria de Gomalcalco

Licenciatura en Enfermería

Licenciatura en Médico Cirujano

Licenciatura en Atención Prehospitalaria y Desastre

Licenciatura en Rehabilitación Física
Divisiőn Acadẻmica de Educaciőn y Artes

Licenciatura en Ciencias de la Educación

Licenciatura en Comunicación

Licenciatura en Desarrollo Cultural (sistema abierto)

Licenciatura en Idiomas

Técnico Superior Universitario en Enseñanza de Inglés

Técnico Superior Universitario en Música

Divisiőn Académica de Ciencias de la Salud

Licenciatura en Médico Cirujano

Licenciatura en Cirujano Dentista

Licenciatura en Psicología

Licenciatura en Nutrición

Licenciatura en Enfermería

Enfermería (semipresencial) Nivel Técnico

Curso complementario de Licenciatura en Enfermería

(a distancia y semipresencial)

\section{Divisiő́n Académica de Informática y Sistemas}

Licenciatura en Informática Administrativa

Licenciatura en Sistemas Computacionales

Licenciatura en Telemática

Licenciatura en Tecnologías de la Información

\section{Divisiő́n Académica de Clencias Bảsicas}

Licenciatura en Ciencias Computacionales

Licenciatura en Física

Licenciatura en Matemáticas

Licenciatura en Química

Licenciatura en Actuaría

Divisiơn Académica Multidisciplinaria de los Ríos

Ingeniería en Acuacultura

Licenciatura en Administración

Ingeniería en Alimentos

Licenciatura en Informática Administrativa

Licenciatura en Derecho 